\title{
ALGUNAS PECULIARIDADES DE LA TRADUCCIÓN DE LAS RIMAS DE BECQUÉR AL GEORGIANO (UN INTENTO DE APROXIMAXIÓN DE DOS CULTURAS)
}

\section{SOME PECULIARITIES OF THE TRANSLATION OF RIMAS DE BÉCQUER (THE INTENT OF THE APPROXIMATION OF TWO CULTURES)}

Vladimer Luarsabirshvili

New Vision University (Tbilisi, Georgia)

\section{ABSTRACT}

The aim of our paper is to investigate some characteristics of literary translations of the texts of Spanish romantic author Gustavo Adolfo Bécquer and to demonstrate and provide arguments of using special translations strategies during translations of Bécquer's Rimas.

Keywords: Culture, translation, Rimas, Bécquer, Georgian.

\section{RESUMEN}

El propósito de nuestro trabajo es tratar algunas peculiaridades de la traducción literaria del texto romántico español y de Gustavo Adolfo Bécquer en particular; mostramos y argumentamos las técnicas usadas en el proceso de traducción de Rimas de Bécquer al 
georgiano, destacamos las dificultades que acompañaron al proceso y proponemos las vías de actividad traductora orientadas a una recepción relevante del texto.

Palabras clave: Cultura, traducción, Rimas, Bécquer, Georgiano.

Fecha de recepción: 15 de octubre de 2019.

Fecha de aceptación: 18 de octubre de 2019.

Cómo citar: Luarsabishvili, Vladimer (2019): «Algunas peculiaridades de la traducción de las Rimas de Bécquer al georgiano (un intento de aproximación de dos culturas», en Actio Nova: Revista de Teoría de la Literatura y Literatura Comparada, 3: 384-406.

DOI: https://doi.org/10.15366/actionova2019.3.016 


\section{INTRODUCCIÓN Y OBJETIVOS}

El propósito de nuestro trabajo es tratar algunas peculiaridades de la traducción literaria del texto romántico español y de Gustavo Adolfo Bécquer en particular; mostramos y argumentamos las técnicas usadas en el proceso de traducción de Rimas de Bécquer al georgiano, destacamos las dificultades que acompañaron al proceso y proponemos las vías de actividad traductora orientadas a una recepción relevante del texto.

Nuestro artículo consta de seis partes. En la primera hacemos una reseña panorámica de la historia de la traducción; en la segunda recordamos brevemente las peculiaridades de la traducción literaria; en la tercera describimos las ediciones de los textos de Bécquer en georgiano; en la cuarta mostramos las técnicas utilizadas en el proceso de traducción de Rimas, en la quinta hacemos una reflexión sobre las traducciones realizadas, y, en la sexta presentamos las conclusiones generales y proponemos las vías de la actividad traductora que persigue el fin de recibir un texto relevante y adecuado.

Como material para nuestra investigación hemos elegido las Rimas XIII, XXX, XXXI, XXXIV, LXVIII, XLII de Bécquer¹.

\section{Historia BREVE DE LA TRADUCCIÓN}

La traducción ha sido un asunto rigurosamente estudiado tanto por los profesionales como por los aficionados de la profesión. Los habitantes del antiguo imperio de Egipto, así como los griegos y los romanos expresaban la relación distinta por el asunto. Los debates sobre la distinción entre las técnicas traductoras las inauguraron Cicerón ${ }^{2}$,

\footnotetext{
${ }^{1}$ Seguiremos, para las citas de la obra de Bécquer, la siguiente edición: Bécquer, Gustavo Adolfo (2003): Rimas y Leyendas, Madrid, Alba.

${ }^{2}$ Necconverti ut interpres, sed ut orator, sententiisisdem et earumformistamquamfiguris, verbis ad nostramconsuetudinemaptis. In quibus non verbum pro verbo necesse habui reddere, sed genus omne verborum vimque servavi. [«And I did not translated them as an interpreter, but as an orator, keeping the same ideas and forms, or as one might say, the 'figures' of thought, but in language which conforms to our usage. And in so doing, I did not hold it necessary to render word for word, but I preserved the general style and force of the language». Cicero, M.T. (46BCE/1960 CE)] 'De optimogenereoratorum', in CiceroDeinventione, De optimogenereoratorum, topica, translated by H.M.Hubbell, Cambridge, MA: Harvard University Press; London: Heinemann: 347-73, citado en Munday, 2008: 19.
} 
Horacio $^{3}$ y San Jerónimo ${ }^{4}$. Distintos métodos de traducción fueron cultivados durante el período Abbāsid (750-1250), y en Toledo del siglo XII funcionaba la escuela de traductores. En el siglo XVII aparecen trabajos de Denham, ${ }^{5}$ Cowley $^{6}$ y Dryden. Los estudiosos alemanes intervinieron en el proceso con las obras de Goethe y Schleiermacher y basaron en la obra del último sus propias investigaciones K. Reiss y L. Venuti.

Veamos sucintamente la parte de la historia de la traducción que está relacionada con el tema de nuestra investigación.

\subsection{LAS CARACTERÍSTICAS CULTURALES E IDEOLÓGICAS}

En su libro de ensayos Susan Bassnet y André Lefevere se alejaron de las teorías lingüísticas de la traducción que «have moved from Word to text as a unit, but not beyond» (Bassnet and Lefevere, 1990: 4). Los autores rechazan el método de la comparación entre el texto y su traducción fuera del contexto cultural e investigan la influencia de la cultura sobre la traducción. La observación de la traducción en el contexto político-cultural y fuera del contexto textual lo llamó Mary Snell-Hornby «the cultural turn». ${ }^{7}$ Bassnett y Lefevere utilizan el término como metáfora para un estudio de los cambios culturales en la traducción.

A la traducción como re-escritura dedica sus estudios A. Lefevere basando sus propias investigaciones en los trabajos de la Escuela de Manipulación y en la teoría de polisistema ${ }^{8}$. El autor se centra en el examen de los factores concretos como fuerza, ideología y manipulación y observa el papel de los representantes de estos factores en la re-escritura de los textos traducidos. La motivación, según él, puede ser determinada por razones ideológicas

\footnotetext{
3 Sobre las ideas de Cicerón y Horacio: García Yebra, Valentín (1979): «¿Cicerón y Horacio preceptistas de la traducción?» en Cuadernos de la filología clásica, 16: 139-154.

4 Ego enim non solumfateor, sed libera voceprofiteor, $m e$ in interpretationeGraecorum, absquescripturissanctis, ubi et verborum ordo et misteriumest, non verbum e verbo, sed sensumexprimere de sensu. [ Now I not only admit but freely announce that in translation from the Greek - except of course in the case of the Holy Scripture, where even the syntax contains a mystery - I render not word-for-word, but sensefor-sense.] Jerome, E.H. (St Jerome) (395 CE/1997) 'De optimegenereinterpretandi' (Letter 101, to Pammachius), in Epistolae D. HieronymiStridoniensis, Rome: Aldi F., (1565), pp. 285-91, translated by P.Carrol as 'on the best kind of translator', in D.Robinson (ed.) (1997b), pp. 22-30, citado en Munday, 2008: 20.

5 (Apud Hervás Jávega, 1998: 263).

${ }^{6}$ (Apud Munday, 2008:25).

${ }^{7}$ Snell-Hornby, Mary (1990): «Linguistic transcoding or cultural transfer: a critique of translation theory in Germany», en S. Bassnett and A. Lefevere (ed), Translation, History and Culture, London and New York, Routledge: 79-86.

${ }^{8}$ Lefevere, André (1992): Translation, rewriting and the Manipulation of Literary Fame, London and New York, Routledge.
} 
(dependientes de la ideología dominante) o poéticas (dependientes de la poética dominante). La ideología limita también la selección de texto traducido, y del componente económico depende la motivación del traductor. No hay que disminuir su papel de crítico literario cuya estimación también facilita la recepción o el rechazo del texto traducido.

Al papel del traductor en el proceso de la traducción le dedica su investigación Lawrence Venuti. Acuñando el término «invisibility», Venuti describe la condición del traductor y su actividad en la literatura contemporánea Anglo-americana:

\begin{abstract}
by the way translators themselves tend to translate 'fluently' into English, to produce an idiomatic and 'readable' TT, thus creating an «illusion of transparency»;

by the way the translated texts are typically read in the target culture:

A translated text, whether prose or poetry, fiction or non-fiction, is judged acceptable by most publishers, reviewers and readers when it reads fluently, when the absence of any linguistic or stylistic peculiarities makes it seem transparent, giving the appearance that it reflects the foreign writer's personality or intention or the essential meaning of the foreign text - the appearance, in other words, that the translation is not in fact a translation, but the 'original' (Venuti, 1995: 1).
\end{abstract}

Junto con el término «invisibility» Venuti ofrece dos métodos de traducción: la domesticación y la extranjerización. Observa la domesticación como «an ethnocentric reduction of the foreign text to [Anglo-American] target-language cultural values» (Venuti, 1995: 20) y la extranjerización que «entails choosing a foreign text and developing a translation method along lines which are excluded by dominant cultural values in the target language» (Venuti, 1998: 242).

La relación de la visión de Venuti con el entendimiento de la estrategia traductora de Antonie Berman es innegable. Berman reflexiona sobre «the experience of foreign»y describe un «épreuve» desde dos perspectivas: «a trial for the target culture in experiencing the strangeness of the foreign text and word; a trial for the foreign text in being uprooted from its original language context» (Berman, 2004: 276).

$\mathrm{Al}$ sistema de la deformación textual durante la traducción Berman lo denomina «negative analytic»y defiende su carácter con las siguientes palabras: «The negative analytic is primarily concerned with ethnocentric, annexationist translations and hypertextual translations (pastiche, imitation, adaptation, free writing), where the play of deforming forces is freely exercised» (Berman, 2004: 278). 


\section{LAS PECULIARIDADES DE LA TRADUCCIÓN LITERARIA}

Los vínculos entre la literatura y la traducción pueden ser observados desde los tiempos antiguos indicando un papel de la traducción en la formación de los textos y géneros literarios (Lambert, 1980; Torre, 1994). Efectivamente, teniendo en cuenta las peculiaridades que ha de poseer el traductor literario, la influencia del proceso en el desarrollo de la literatura meta es inevitable. Por una parte, Octavio Paz subrayó la dificultad que posee un traductor literario al no tener las mismas posibilidades creativas del autor de texto original (1971); por otra, un traductor literario ha de poseer una cierta competencia literaria (van Dijk, 1976; Aguiar e Silva, 1977; García Berrio, 1994). Debe poseer, además, competencias lingüísticas, literarias y técnicas especiales (García Yebra, 1982: 30-33). El profesor Antonio García Berrio propuso el concepto de práctica sistemática de la excepción comunicativa tratando el asunto de la libertad del traductor literario (1994: 81-97) y el profesor Tomás Albaladejo indica que, por su naturaleza, la traducción literaria «es una reescritura, una forma de creación, que permite que pueda llegar a una intervención con interferencia creadora en los diferentes niveles de la obra, aunque con el mantenimiento del significado o, al menos, de lo esencial de éste». (2001:

5). Entonces es necesario que el traductor literario posea una «competencia lingüística pasiva de la lengua de partida y competencia lingǘstica activa de la lengua de llegada, aunque el grado ideal de competencia lingüística del traductor es el que se da cuando éste posee competencia tanto activa como pasiva de ambas lenguas» (Albaladejo, 1992: 182). Según el profesor Francisco Chico Rico «El traductor literario, en su actividad global, produce o construye los elementos semántico-extensionales y sintáctico-semióticos de la obra de arte verbal terminal en la medida en que los ha construido el autor de la obra de arte verbal original» (2001: 267).

La relación entre el texto traducido y el lugar que éste ocupa en la literatura meta es tan importante, que el profesor israelí Itamar Even-Zohar propuso una teoría de polisistema (Even-Zohar, 2004) según el que la literatura traducida puede ocupar un lugar primario o secundario en el sistema literario. La interacción entre la literatura y la traducción sigue siendo importante hasta nuestros días, por el enriqueciendo mutuo y por la facilidad con la que se produce la comunicación (Albaladejo, 2007: 61). Relacionada con la semiótica, la traducción participa en la transformación de un tipo de mensaje en otro tipo de mensaje (Jakobson, 
1975), desarrollada en un espacio semiótico (Morris, 1971; Bobes Naves, 1973), al mismo tiempo siendo un fruto del mismo proceso (García Yebra, 1982). Reflexionando sobre la tradición histórica de la traducción, el profesor Tomás Albaladejo Mayordomo indica la función dinamizadora que cumple en el proceso de la comunicación (Albaladejo, 2007: 61). En una serie de trabajos, a partir de la teoría de la estructura del texto y de la estructura del mundo de János S. Petöfi $(1971,1973,1975,1979,1992)$, el profesor ha construido el modelo textual de traducción literaria (Albaladejo, 1981, 1982, 1992). Siendo una forma de transducción (Doležel, 1986), el texto traducido necesita pasar no sólo por el proceso de cambios del estado original, sino mantener tanto la estructura como el mensaje semántico de la cultura de partida (Albaladejo, 2004). Como actividad interdiscursiva, la traducción es una fuente para el nacimiento de un texto nuevo y aquí aparece el papel que puede desempeñar la intertextualidad en el proceso de traducción (Sánchez García, 1996). Efectivamente, se empieza a estudiar el papel del intertexto de época en el proceso de traducción (Luarsabishvili, 2013).

\section{TEXTOS DE BÉCQUER EN GEORGIANO}

En el proceso de aproximación de culturas, el proceso de traducción desempeña un papel importante si no decisivo 9 . Durante el siglo XX los textos de varios autores españoles han sido traducidos al georgiano. Haciendo una búsqueda en el sistema electrónico del catálogo de la Biblioteca Nacional de Georgia ${ }^{10}$, encontramos la lista de los libros traducidos.

A pesar de que la lista no es corta (algunos 60 títulos), todavía nos faltan investigaciones del ámbito traductológico español-georgiano que puedan arrojar luz sobre las técnicas utilizadas durante la traducción, las estrategias usadas en casos de los textos

\footnotetext{
${ }^{9}$ Sobre la aproximación de las culturas mediante la traducción: Kasperska, Iwona (2012): «Traducción del nuevo mundo: ¿Diálogo intercultural o confrontación de culturas? Aproximación a la visión del otro en las crónicas del descubrimiento y la conquista», en Studia Romanica Posnaniensa, UAM, 39/2: 23-39, Lvovskaya, Zinaida (1999): «Intertextualidad cultural en traducción», en Actas de la II conferencia de hispanistas de Rusia (Moscú, 1923 de abril de 1999). Madrid, Embajada de España en Moscú; Bogomilova Alanassova, Denitsa (2004): «Un enfoque hermenéutico de la traducción cultural», en Hermèneus. Revista de Traducción e Interpretación, 6: 19-39; Gogazeh, Ziyad (2005): «Problemas culturales y lingüísticos en la traducción de refranes del árabe al español y viceversa», en Paremia, 14: 61-70.

10 http://www.nplg.gov.ge/eng/home.
} 
pertenecientes a diferentes épocas literarias ${ }^{11}$. La reflexión sobre las vías concretas de la traducción puede facilitar, a nuestra forma de ver, el proceso de entendimiento del problema traductológico y a la aproximación de dos culturas tan diferentes.

Intentando reflexionar sobre las dificultades concretas que pueden acompañar al proceso de traducción español-georgiana, no hay que olvidar que cierta cantidad de traducciones no incluyen la leyenda que indica la lengua de partida, es decir desde la cual fue traducida la obra concreta. Esto es muy importante puesto que en Georgia la lengua española no fue muy difundida y los traductores han realizado sus traducciones desde la lengua dominante de la Unión Soviética, es decir, del ruso. Además de la dificultad de la diferenciación de estas traducciones de las realizadas desde la lengua original, estos casos deben ser excluidos tratando las características de la traducción español-georgiana.

\section{LAS TÉCNICAS UTILIZADAS DURANTE LA TRADUCCIÓN DE LAS RIMAS}

Antes de entrar en el tema hay que mencionar que las traducciones de las Rimas de Bécquer no pueden ocupar una posición primaria en el polisistema de la literatura georgiana pues:

a) la literatura georgiana no es $\left\langle\right.$ joven $»^{12}$;

b) no está «periférica»o «débil» para que sea necesario importar formas literarias nuevas;

c) no se encuentra en una etapa crítica de su desarrollo.

De manera que, es evidente que, según la Teoría de los polisistemas, las traducciones de las Rimas ocuparán la posición segunda y por eso pueden ser tratadas como «norma» según la definición de Even-Zohar. Añadimos, que realizando las traducciones al georgiano hemos elegido la técnica de extranjerización porque: a) intentamos destacar las características lingüístico-culturales del texto ajenas para la cultura meta (georgiano, en nuestro caso), y, b) deseamos buscar un lugar periférico para las traducciones en la literatura georgiana que permita conservar las peculiaridades culturales del original en la lengua meta.

${ }^{11}$ Sobre el tema de la traducción literaria de los textos románticos: Luarsabishvili, Vladimer (2012): «Un intento de la traducción poética: Gustavo Adolfo Bécquer en georgiano (algunos ejemplos)», en Hermèneus, 14: 363368; Luarsabishvili, Vladimer (2014): «Retórica y traducción: las rimas de Bécquer en georgiano», en Rétor, Revista de Retórica, 4, 1: 36-55.

${ }^{12} \mathrm{El}$ primer texto literario georgiano es del siglo V («El martirio de la reina Santa Shushanik»). 
Junto con la teoría de Venuti, la estrategia traductora elegida por nosotros está basada en la teoría de Berman y su sistema de deformación textual en particular. Con esto, hemos eludido un fenómeno de naturalización para que el lector reciba lo ajeno como ajeno, sin domesticarlo.

Leemos la rima XII:

Tu pupila es azul, y cuando ríes, su claridad suave me recuerda el trémulo fulgor de la mañana que en el mar se refleja.

Tu pupila es azul, y cuando lloras, las transparentes lágrimas en ella se me figuran gotas de rocío sobre una violeta.

Tu pupila es azul, y si en su fondo como un punto de luz radia una idea, me parece en el cielo de la tarde ¡una perdida estrella!

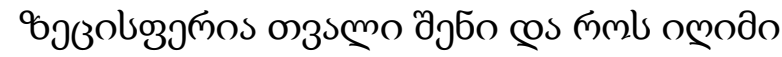

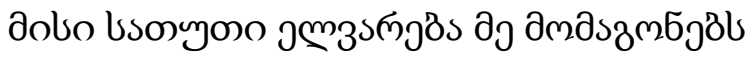

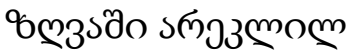

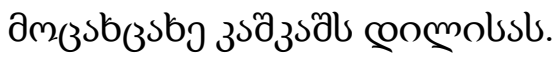

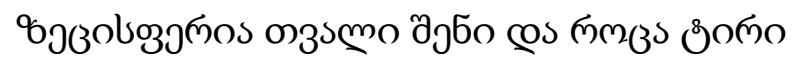

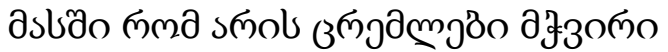

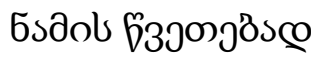

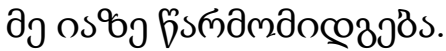




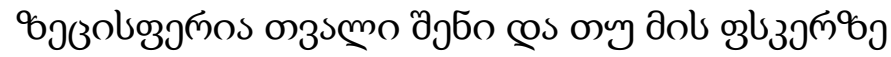

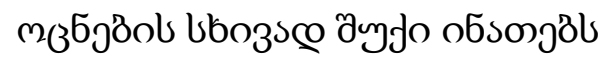

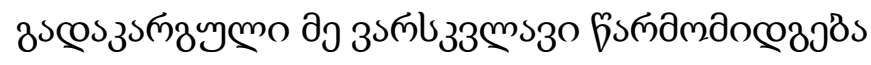 \\ uscsamb 3s\%g.
}

Suscita nuestro interés el hecho que lastres estrofas empiezan de manera igual: «Tu pupila es azul», pero en la primera estrofa destacamos los verbos oposicionales - reir-llorar. En la traducción hemos guardado la estructura y traducimos «Tu pupila es azul...ríes» como

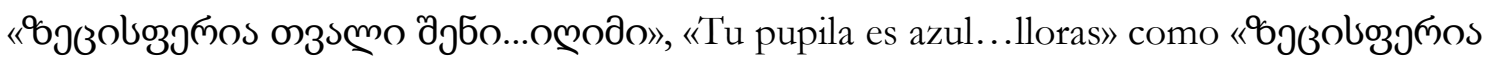

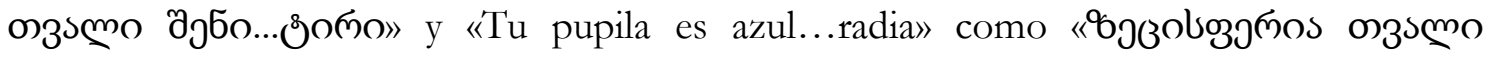

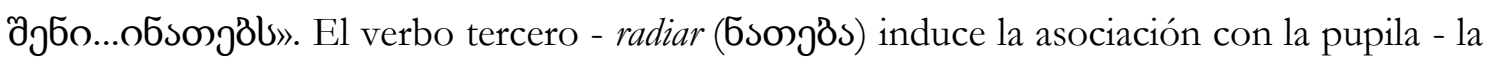
luz, el ver, etc. La risa (ભুodomo) está asociada con «el trémulo fulgor de la mañana» (coomols

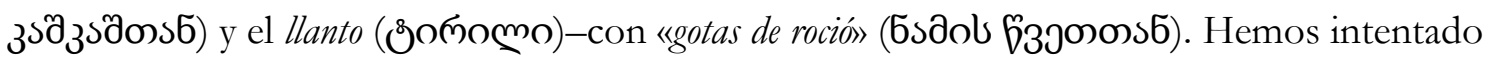
conservar las comparaciones y «el trémulo fulgor de la mañana» lo hemos traducido como

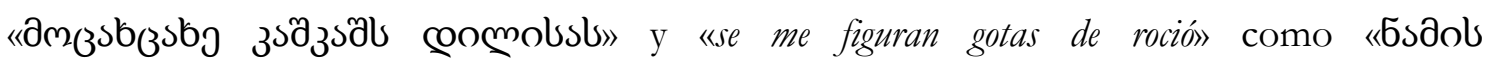

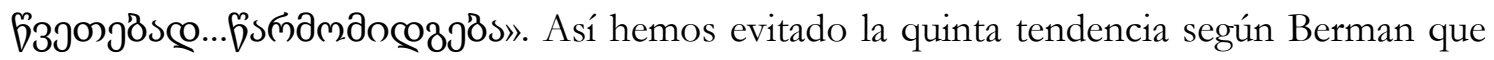
se llama empobrecimiento/agotamiento cualitativo (qualitative impoverishment) y en el texto traducido no se ha perdido la semántica original. En otras palabras, hemos encontrado la equivalencia en la lengua meta que por una parte guarda la riqueza del sonido y, al mismo tiempo, no pierde su significado. En lo que se refiere a la tercera estrofa, en la traducción hemos guardado tanto las características semánticas del texto original, como el mensaje emocional, lo que ha hecho posible la transferencia de algunas metáforas del romanticismo (llorar, estrella perdida).

Leemos la rima XXX:

Asomaba a sus ojos una lágrima

y a mi labio una frase de perdón:

habló el orgullo y enjugó su llanto,

y la frase en mis labios expiró. 
Yo voy por un camino, ella por otro;

pero al pensar en nuestro mutuo amor,

yo digo aún: «Por qué callé aquel día?»

y ella dirá: «Por qué no lloré yo?»

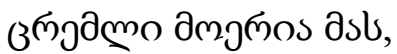

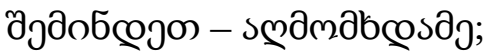

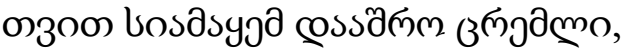

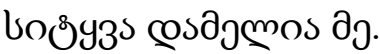

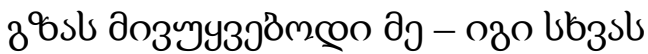

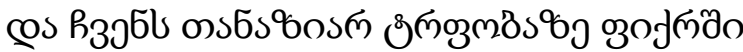

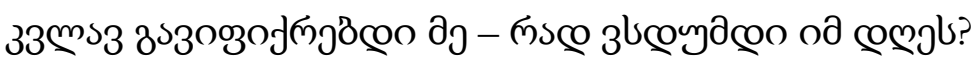

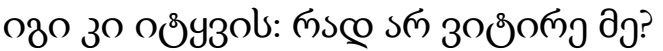

Observamos en esta rima un cambio de papeles románticos entre el poeta y la amada, cuando la emoción del poeta (en nuestro caso -lágrima) la expresa la mujer y la expresión ajena para el poeta-verbal (y no emocional) la expresa el mismo poeta. En el texto original, en la primera estrofa está subrayado el fin de ambas expresiones -emocional y verbal

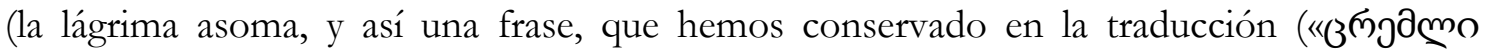
дмэґо según Berman - racionalización que se trata de la estructura y secuencia de la oración. Transfiriendo ambos factores, hemos guardado el mensaje semántico-emocional del texto original en la lengua meta.

Leemos la rima XXXI:

Nuestra pasión fue un trágico sainete,

en cuya absurda fábula

lo cómico y lo grave confundidos

risas y llanto arrancan. 
Pero fue lo peor de aquella historia,

que, al fin de la jornada,

a ella tocaron lágrimas y risas,

¡y a mí sólo las lágrimas!

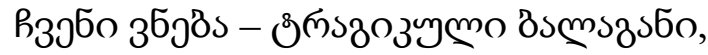

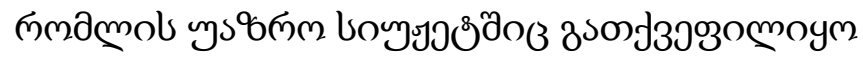

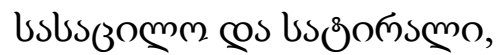

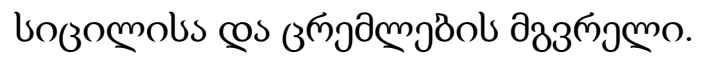

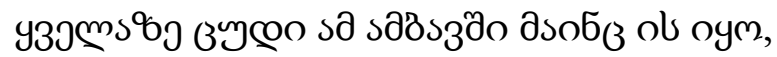

пма ymozol ठ̀mलmmb

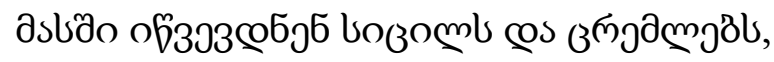

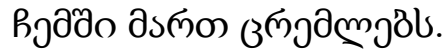

Una atención especial merecela traducción de los miembros del binomio risas-llanto que se modulan uno a otro, caso que Iuri Lotman denominó «los casos más complicados» ${ }^{13}$. Esto posee importancia, además, desde un punto de vista de la quinta tendencia de Berman - agotamiento cualitativo (qualitative impoverishment) - en el caso de reemplazo de algún miembro del binomio (incluyendo los sinónimos) es posible recibir la destrucción del mensaje semántico-emocional del texto original. Pensamos que no fueron elegidas por Bécquer las metáforas de llanto, risa y lágrima por casualidad porque ellas poseen, junto con el significado primero, también los significados secundarios, es decir son, conceptos. Por eso conservamos las palabras mencionadas y no las cambiamos con sus equivalentes.

El único cambio que hemos realizado fue la traducción de la palabra «historia» como «ymojs». En la lengua georgiana, esta palabra posee un significado más amplio que

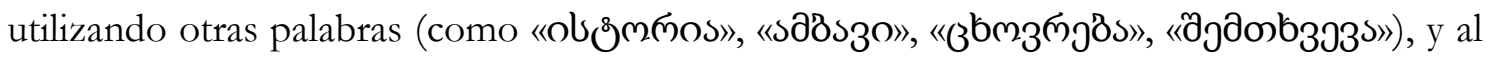


mismo tiempo, expresa la elevación sobre la objetividad, que es una de las características de la ironía romántica ${ }^{14}$.

Leemos la rima XXXIV:

Cruza callada, y son sus movimientos

silenciosa armonía;

suenan sus pasos, y al sonar, recuerdan

del himno alado la cadencia rítmica.

Los ojos entreabre, aquellos ojos

tan claros como el día;

y la tierra y el cielo, cuanto abarcan,

arden con nueva luz en sus pupilas.

Ríe, y su carcajada tiene notas

del agua fugitivo;

llora, y es cada lágrima un poema

de ternura infinita.

Ella tiene luz, tiene el perfume,

el calor y la línea;

la forma engendradora de deseos,

la expresión, fuente eternal de poesía.

¿Qué es estúpida?... ¡Bah! Mientras callando

guarde oscuro el enigma,

siempre valdrá, a mi ver, lo que ella calla

más que lo que cualquiera otra me diga.

${ }^{14}$ Sobre la ironía romántica en las Rimas de Bécquer: Luarsabishvili, Vladimer (2012): «La ironía romántica en las Rimas de Bécquer», en AdversuS, IX, 22: 136-149. 
coscoub ybosyzmç, дoдmb3ms dobo

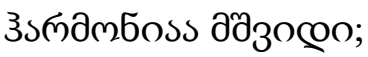

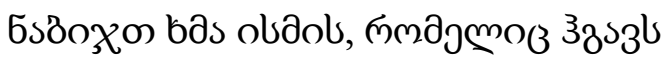

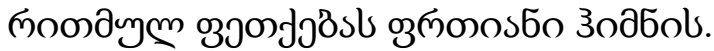

comjlszon 6songerl

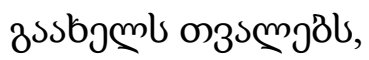

cs dof̧s cos zs hsbyóg'oymbo

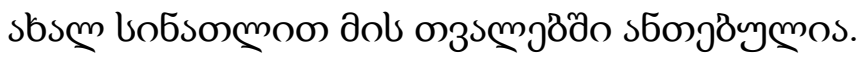

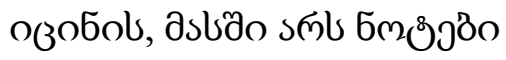

8stojymo fymol,

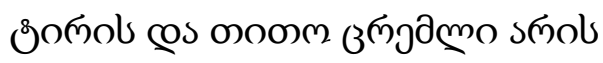

эзосозьбм bo5s\%ol maflo.

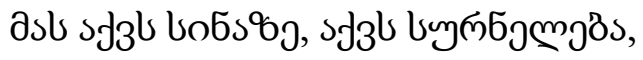

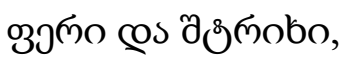

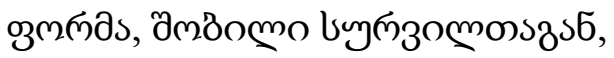

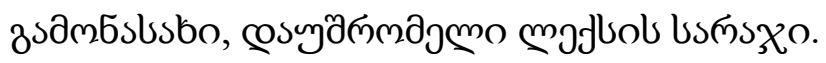

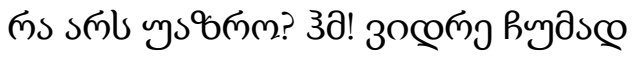

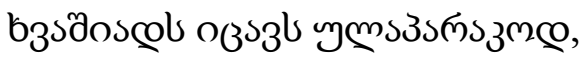

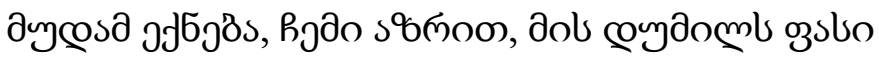

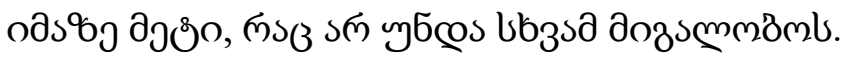

Hemos conservado en la traducción los miembros mutuo-analógicos ríe-llora y notas-

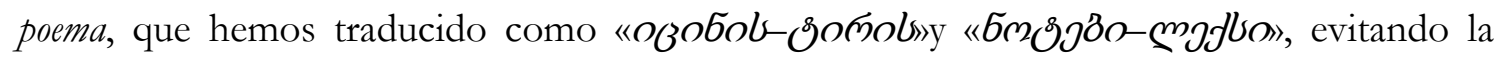
quinta tendencia según Berman. Al mismo tiempo, la metáfora universal del romanticismo 


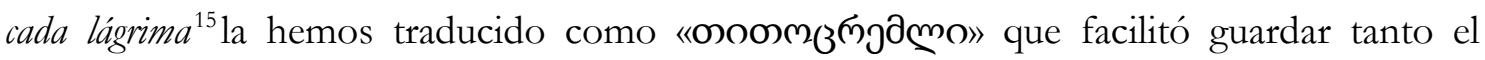
mensaje semántico como cultural. Hemos aludido a la «reducción etnocéntrica» del texto ajeno por la cultura meta (por la cultura georgiana en nuestro caso). Para L. Venuti aquel factor es decisivo. En caso de la técnica de domesticación el texto traducido no puede resistir la influencia de las normas literarias georgianas lo que podría resultar en la pérdida de las

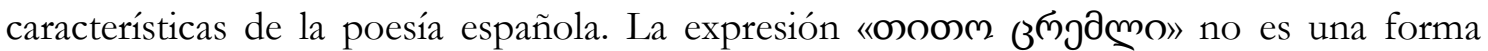
literaria aplicada en la literatura georgiana, pero su utilización ayuda a conservarlas características de la cultura original en la cultura meta.

Leemos la rima LXVIII:

No sé lo que he soñado

en la noche pasada;

triste, muy triste debió ser el sueño

pues despierto la angustia me duraba.

Noté, al incorporarme,

húmeda la almohada,

y por primera vez sentí, al notarlo,

de un amargo placer henchirse el alma.

Triste cosa es el sueño

que llanto nos arranca;

mas tengo en mi tristeza una alegría...

¡sé que aún me quedan lágrimas!

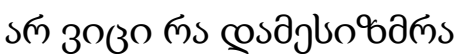

gsturgm nsajl,

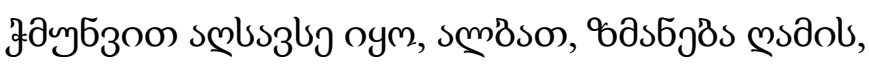

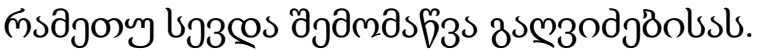

15Sobre la cosmovisión romántica: Sebold, Russel P. (2011); «La cosmovisión romántica: siete síntomas y cinco metáforas», en Castilla. Estudios de Literatura, 2: 311-323. 


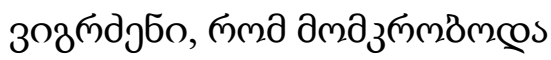

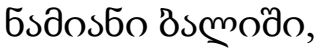

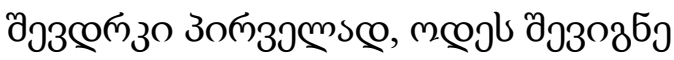

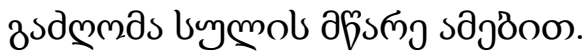

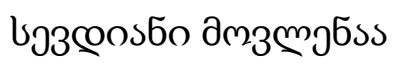

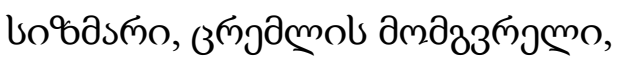

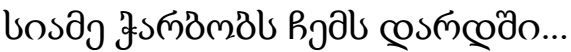

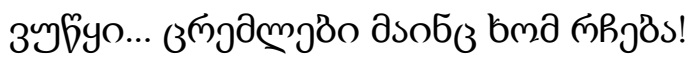

La culminación semántica de esta rima está encerrada en su tercera estrofa. Por una parte, en el primer plano vemos la metáfora universal del romanticismo - la lágrima («que llanto nos arranca»), por otra -indica el poeta la presencia de la alegría en su tristeza («mas tengo en mi tristeza una alegría») que nos recuerda la ética kantiana (las tres preguntas famosas), el existencialismo unamuniano (el dolor como un camino para la conciencia) y un síntoma universal del romanticismo (el goce en el dolor ${ }^{16}$. Basándonos en lo mencionado, fue muy importante guardar este mensaje semántico en la traducción -traducimos «que llanto nos arranca» como «з

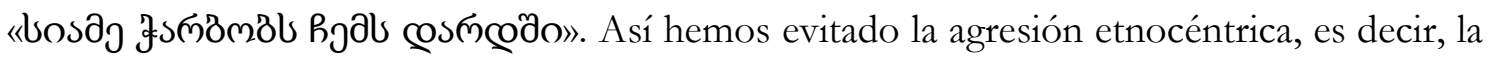
influencia de la cultura meta sobre el texto original.

Leemos la rima XLII:

Cuando me lo contaron sentí el frio de una hoja de acero en las entrañas; me apoyé contra el muro, y un instante la conciencia perdí de donde estaba.

Cayó sobre mi espíritu la noche:

en ira y en piedad se anegó el alma... 
¡Y entonces comprendí por qué se llora,

y entonces comprendí por qué se mata!

Pasó la nube de dolor... con pena

logré balbucear breves palabras...

¿Quién me dio la noticia?... Un fiel amigo...

¡Me hacía un gran favor!... Le di las gracias.

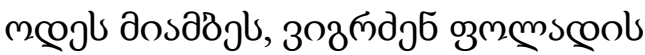

loзозэ дозбоо,

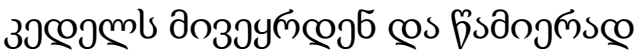

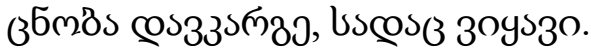

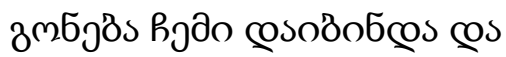

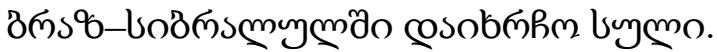

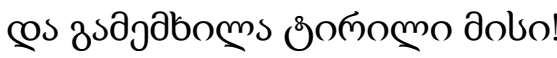

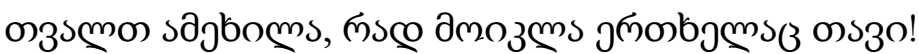

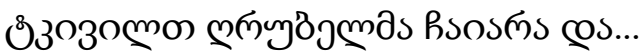

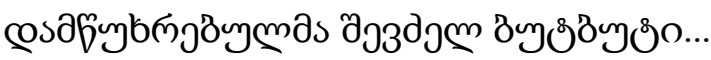

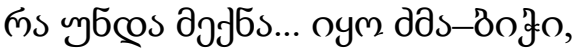

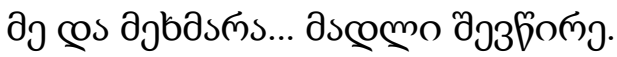

He aquí las tres expresiones de la estética romántica, a las que hemos prestado atención especial durante la traducción: a) la tercera línea de la segunda estrofa («Y entonces comprendí por qué se llora, »), b) la modalidad de llanto como uno de los elementos kinésicos, y c) la metáfora universal del romanticismo (lágrima/llanto). Muy importante también fue guardar la expresión hegeliana -«y entonces comprendí por qué se mata». Hemos traducido «Y entonces comprendí por qué se llora» como «@s zsajaboms s̊on̆omo dobo» y «y

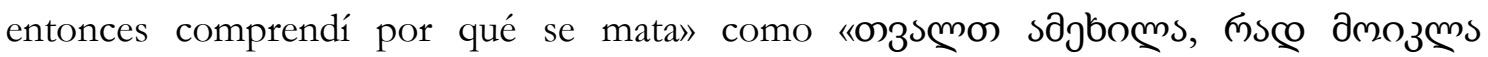




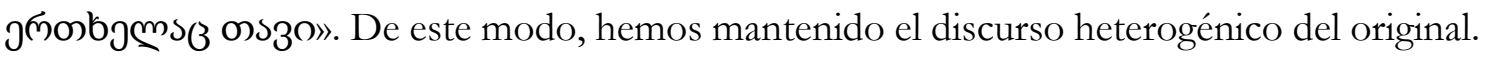
La técnica de extranjerización nos permite, por una parte, guardar en la traducción las características de la cultura original, y, por otra, no perder el significado de las metáforas y síntomas universales del romanticismo.

\section{LA REFLEXIÓN SOBRE EL TRABAJO REALIZADO}

Para realizar un intento de traducción de las Rimas de Bécquer al georgiano, hemos basado nuestro trabajo en ciertas estrategias recomendadas por distintos autores. Entre ellos, destacan los trabajos de Antonie Berman, André Lefevere and Lawrence Venuti.

Efectivamente, para nosotros fue importante evitar las tendencias deformantes que describe Berman en su famoso texto. Leyendo y revisando las traducciones realizadas por los traductores georgianos, observamos sus conocimientos de las técnicas de la traducción difundidas en los siglos pasados. Y, al contrario, investigando las traducciones realizadas en el siglo XX, se presenta ante nosotros la ignorancia completa de las teorías del siglo pasado. En particular, a la mayoría de los traductores les falta no sólo el conocimiento de la teoría de Berman, sino también de la de Lefevere y Venuti. Explicamos brevemente a lo que nos referimos.

Hablando sobre los factores que influyen en la aceptación de los textos traducidos, Lefevere sobreentiende las instituciones como el poder, la ideología y la manipulación. La elección del texto que debe ser traducido depende de la poética o ideología dominante. Teniendo en cuenta también los factores políticos, no hay que olvidar que durante casi un siglo Georgia formó parte de la Unión Soviética, lo que influyó no sólo en la existencia económica y política del país, sino en la formación de la mentalidad de sus ciudadanos. Es bien sabido, que en los países totalitarios la literatura estaba al servicio del poder $^{17}-$ lo que fue admitido en la Rusia soviética, fue difundido en otras repúblicas soviéticas y Georgia no fue la excepción. Basándose en los asuntos de ideología, en la Unión Soviética fueron ampliamente traducidos los textos de Federico García Lorca y Thomas Mann, como víctimas del fascismo. Junto con

\footnotetext{
${ }^{17}$ Sobre el tema: Policinska, Marta (2008): «La literatura al servicio del estado: algunas consideraciones sobre la utilización propagandística de la literatura en la Unión Soviética de los años 20 y 30», en Comunicación, 1, 6: 118129; Suárez López-Zuriaga, Eloísa (2008): «Estalinismo y religión Política: entre la ficción y los acontecimientos históricos», en Bajo Palabra, Revista de Filosofía, 3: 165-172.
} 
la ideología, desempeñó un papel importante la poética dominante - traducir la poesía del mismo Lorca utilizando las técnicas de la expresión vanguardista no podría resultar en la creación de una traducción aceptable. De esta manera, en la Unión Soviética se formó una estrategia de producción de texto aceptable, o utilizando un término de Venuti-domesticado. Como ejemplo de lo dicho nos pueden servir no sólo las traducciones de Lorca realizadas por la poetisa rusa Marina Tsvetaeva, sino también las de los sonetos de Shakespeare realizadas por Samuil Marshak ${ }^{18}$. Lo contrario ha hecho L. Venuti que ha traducido los textos del autor del siglo XIX Iginio Ugo Tarchetti, manteniendo el estilo del autor y traduciendo sus textos con un vocabulario arcaico ${ }^{19}$.

\section{CONCLUSIONES FINALES}

La estrategia que elige un traductor desempeña un papel decisivo para la recepción de un producto traductológico relevante. Ella puede ser determinada teniendo en cuenta los distintos factores que influyen sobre su elección (Ideología, poética dominante, situación política y económica en el país, tradiciones de la escuela traductológica, entre otros).

La estrategia traductora tiene que ser correspondiente con el texto y la época concreta tanto de la cultura original como de la cultura meta. Cada época literaria posee su vocabulario, formas de expresión (síntomas, metáforas) y visión del mundo especial, lo que debe ser en correspondencia en cada caso concreto.

La técnica de la domesticación ayuda a guardar tanto las características semánticoculturales, como emocionales del texto original. La fidelidad del traductor al autor es una condición sine que non para recibir una traducción relevante.

\footnotetext{
${ }^{18}$ Sobre el tema: Gasparov, Mikhail, Avtonomova Nataliya (2001): Soneti Shekspira - perevodi Marshaka, en Gasparov M.L., O russkoi poeziii, Petersburgo.

${ }^{19}$ Sobre el tema: Iginio Ugo Tarchetti, The Fantastic Tales, translated by Lawrence Venuti, Alma Books, 2013: 224.
} 
BIBLIOGRAFÍA

Aguiar e Silva, Vítor Manuel de (1977): Competência lingüistica e competencia literaria, Coimbra, Almedina.

Albaladejo Mayordomo, Tomás (1981): «Aspectos del análisis formal de textos», en Revista Española de Lingüistica, 11: 117-160.

Albaladejo Mayordomo, Tomás (1987): «Componente pragmático, componente de representación y modelo lingüístico-textual», en Lingua e Stile, 18, 1: 3-46.

Albaladejo Mayordomo, Tomás (1992): «Aspectos pragmáticos y semánticos de la traducción del texto literario», en KOINÉ. Annali della Scuola Superiore per Interpreti e Traduttori «San Pellegrino», II, 1-2: 179-200.

Albaladejo Mayordomo, Tomás (2001): «Traducción e interferencias comunicativas», en Hermèneus. Revista de Traducción e Interpretación, 3: 39-58.

Albaladejo Mayordomo, Tomás (2004): Similarity and Difference in LiteraryTranslation, en Stefano Arduini y Robert Hodgson (eds.): Similarity and Difference in Translation, Nueva York - Rimini, Nida Institute for Biblical Scholarship, Guaraldi.

Albaladejo Mayordomo, Tomás (2007): Semiótica, traducción literaria y análisis interdiscursivo, en Miguel Ángel Garrido, Emilio Frechilla Díaz (eds.): Teoría/Crítica. Homenaje a la Profesora Carmen Bobes Naves, Madrid, Consejo Superior de Investigaciones Científicas.

Bassnet, Susan, Lefevere, André (1990), (eds.), Translation, History and Culture. London and New York, Pinter.

Berman, Antoine (2004): «Translation and the trials of the foreign», en L.Venuti (ed.), The Translation Studies Reader, London \& New York, Routledge.

Bobes Naves, Carmen (1973): La Semiótica como teoría lingüística, Madrid, Gredos.

Chico Rico, Francisco (2001): Retórica y traducción. ‘Nóesis’ y ‘Poíesis’ en la traducción del texto literario, en Lenguas, Literatura y traducción. Aproximaciones teóricas, Pierre-Yves Raccah and Belén Saiz Noed (eds.), Madrid, Arrecife.

Dijk, Teun A.Van (1976): Per una poetica generativa, Bolonia, il Mulino.

Doležel, Lubomír (1986): «Semiotics of Literary Communication», en Strumenti Critici, 50, I, 1: $5-48$. 
Even-Zohar, Itamar (2004): The position of translated literature within the literary polysystem, en L.Venuti (ed.), The Translation Studies Reader, (2004): London \& New York, Routledge.

García Berrio, Antonio (1994): Teoría de la Literatura (La construcción del significado poético), Madrid, Cátedra.

García Yebra, Valentin (1982): Teoría y práctica de la traducción, Madrid, Gredos.

Hervás Jávega, Isabel (1998): «La reflexión traductológica: una revisión diacrónica (I) », en Philologia Hispalensis, 12: 225-267.

Jakobson, Roman (1975): En torno a los aspectos lingüisticos de la traducción, en Roman Jakobson, Ensayos de lingüistica general, Barcelona, Seix Barral.

Lambert, José (1980): «Production, tradition et importation: une clef pour la description de la littérature et de la littérature en traduction», en Canadian Review of Comparative Literature/ revue Canadienne de Littérature Comparée, 7 : 246-252.

Luarsabishvili, Vladimer (2013): «Nuevo tipo de intertextualidad: ¿qué es el intertexto de época? El papel del intertexto de época en el proceso de traducción», en Tonos Digital, 24: 119. En https://www.um.es/tonosdigital/znum24/secciones/tritonos-1luarsabishvili_intertexto_de_epoca.htm (último acceso: 20/11/2019).

Morris, Charles (1971): Foundations of the Theory of Signs, Charles Morris: Writings on the General Theory of Signs. La Haya, Mouton, (1971): 13-71.

Munday, Jeremy (2008): Introducing Translation Studies. Theories and applications, London \& New York, Routledge.

Paz, Octavio (1971): Traducción: literatura y literalidad, Barcelona, Tusquets.

Petöfi, János (1971):Transformations grammatiken und eine kotextuelle Text-theorie, Frankfurt, Athenäum.

Petöfi, János (1973): «Towards an Empirically Motivated Grammatical Theory of verbal Texts», en János S. Petöfi - Hannes RIESER (ed.), Studies in text Grammar. Dordrecht, Reidel.

Petöfi, János (1975): Vers une théorie partielle du texte, Papiere zur textlinguistik, Hamburgo, Buske.

Petöfi, János (1979): «Una teoría textual formal y semiótica como teoría integrada del lenguaje natural (Notas metodológicas)», en János S. Petöfi - Antonio García Berrio (ed.), Lingüistica del texto y crítica literaria. Madrid: Comunicación, (1979): 127-145. 
Petöfi, János (1992): «Interpretation and Translating in a Semiotic textological Framework», en Koiné. Quaderni di Ricerca e Didattica sulla Traduzione e l'Interpretaz̧ione, II, 1-2: 263280.

Sánchez García, Jesús (1996): «La comparación intertextual en una aproximación al texto traducido dentro de la traductología descriptiva», en Epos. Revista de Filología, XII: 357-378.

Torre, Esteban (1994): Teoría de la traducción literaria, Madrid, Síntesis.

Venuti, Lawrence (1995): The Translator's Invisibility: A History of Translation, London \& New York, Routledge.

Venuti, Lawrence (1998): «The American Tradition», en M.Baker and Sameh Fekry (ed.) (1998): Routledge Encyclopedia of Translation Studies, London \& New York, Routledge: 30515.

Venuti, Lawrence (2004): The Translation Studies Reader, London \& New York, Routledge. 
SOBRE EL AUTOR

\section{Vladimer Luarsabishvili}

Traductor e hispanista, profesor en la Universidad de New Vision (Georgia). Doctor por la Universidad Autónoma de Madrid, es especialista en Gustavo Adolfo Bécquer y Miguel de Unamuno, ha publicado artículos en las revistas especializadas y ha organizado coloquios internacionales. Sus líneas de investigación básicas son: la traducción literaria, retórica cultural, sistemas totalitarios, teoría de la literatura.

Contact information:

Correo electrónico: vluarsabishvili@newvision.ge 